\title{
Evolution of Telehealth in Ambulatory Psychiatry: A One Year Perspective
}

\author{
Pamela Peck ${ }^{1} \cdot$ John Torous ${ }^{1} \cdot$ Sabra Sullivan ${ }^{1}$ \\ Accepted: 21 June 2021 / Published online: 1 July 2021 \\ (c) The Author(s), under exclusive licence to Springer Science+Business Media, LLC, part of Springer Nature 2021
}

\begin{abstract}
Under the direction of the leadership at our medical center, beginning March 16, 2020, all non-urgent in-person ambulatory visits were to be limited, either rescheduled or performed virtually, as the hospital braced for the surge of COVID-19 patients. The outpatient psychiatry department quickly transitioned to a telehealth model. This paper details our actions taken to implement this plan, reflections on our experience one year later, and areas for future study. On the one-year anniversary of our department implementing remote care practices around COVID-19, we reflect on lessons learned in the transition and maintenance phases of the last 12 months. Reflecting on next steps as a face-to-face care becomes more possible, we share three core factors in our decision making and research opportunities to better quantify the impact of telehealth in 2021 and beyond.
\end{abstract}

Keywords Telehealth · Ambulatory psychiatry · COVID-19

\section{A New Beginning}

A small group of us sat together in our conference room on March 13, 2020. It was coincidentally, Friday the 13th. Our task was to immediately minimize patient and clinician exposure beginning the next week for an uncertain length of time. Like many other practices around the world, 2 weeks later we had converted our clinic to $100 \%$ telehealth (Yellowlees et al., 2020), using video and telephone for new and follow up outpatient appointments, which included psychopharmacology visits as well as psychotherapy and neuropsychology assessment. On that Friday, none of us would have imagined the changes that would be made in response to the biggest global pandemic in our lifetime. 12 months later, we continue to provide the vast majority of our visits via Telehealth.

John Torous

jtorous@bidmc.harvard.edu

Pamela Peck

ppeck@bidmc.harvard.edu

Sabra Sullivan

ssulli1@bidmc.harvard.edu

1 Department of Psychiatry, Beth Israel Deaconess Medical Center, Harvard Medical School, 330 Brookline Ave, Boston, MA 02446, USA
We are a psychiatry ambulatory service in a tertiary care teaching hospital in downtown Boston. Our faculty practice of 20 includes psychiatrists, licensed social workers, and neuropsychologists. In addition, we are an outpatient training site for over 25 psychiatric residents. At least half of the attending group were senior experienced clinicians. Few of our faculty or trainees had ever performed a virtual appointment with a patient and we had no implementation plan for telehealth. We did, however, have an online medical record (OMR) that is both intuitive and able to be accessed remotely. We were embedded in a medical center with an active IT group, a developed compliance department and OMR support. And, most importantly, we had the human capital of engaged and committed clinicians, administrators, and front staff.

\section{The Initial Response}

The overriding priority for us was to ensure uninterrupted safe treatment with our patients. The rapid conversion to online care was a major barrier to creating a research plan or create novel outcomes to assess the transition. Instead, we quickly engaged with our IT and compliance departments to obtain access to a HIPAA approved video platform for virtual care. We reached out to each clinician, to determine 
which patients in their schedule for the next week were acute and needed to be "seen", converting those appointments to video/telephone. Lastly, we contacted our patients to notify them of the immediate changes. Within two weeks, all schedules were up and running via telehealth. At that point, we could not have predicted that one year later over $95 \%$ of our appointments would continue to be through telehealth.

Communication was essential during this transition to bring a dispersed community of clinicians together, provide information in a rapidly evolving system, and most importantly, create a sense that we can do this. The Director sent update emails each weekday, which became the vehicle for the "how tos" of implementation. Each day offered a new operational challenge. The strain of the steep learning curve and the challenge of assessing new and established patients over a phone or video was exhausting. The update emails balanced information with genuine appreciation for the clinicians' effort in the setting of worry about the health of themselves and their loved ones. In 2 weeks, the email updates became weekly. And now, intermittent. Weekly video meetings continued to share both information and experience, and eventually moved to monthly video meetings. We were able to capture quality improvement data through our annual patient satisfaction survey and thus able to assess some aspects of the patient experience of care several months into the "COVID" year. Overall patient satisfaction scores were two points higher compared to the year before COVID-19, reflecting that overall, the transition did not cause a drop on patient perception of care. We were able to use this and other quality metrics to help monitor care. One year later we better recognize a series of new metrics and care makers to study and assess.

\section{Reflections 1 Year Later}

We embraced telehealth out of necessity, responding to the crisis and the mental health surge of the past year. Our total visits for the year prior to the pandemic (2019), were just under 14,000. For the pandemic year (2020), our total visits were nearer to 15,000 . In 2020, we decreased our cancellation/no show rates by over $10 \%$. The increased need for mental health services, the ease of appointments, no travel, and direct outreach by the clinician, contributed to this decrease. By year's end, approximately $65 \%$ of our visits are through video, while the rest are by telephone. We learned lessons about privacy, alliance and clinical care, as well as technology itself over these 12 months and these themes appear in line with the experience of other practices earlier in the pandemic (Uscher-Pines et al., 2020).

Privacy, for patients and clinicians, became an area of creative navigation. The frame of sessions changed. Explicit encouragement by the clinicians for patients to find a quiet space was not enough to keep children, spouses, or pets out of the picture. We were brought on tours of patients' homes, proudly shown awards and grandchildren's pictures as well as shameful acknowledgments of clutter and mess. As clinicians we often hear of the important people in our patients' lives, now we meet a spouse walking by the sofa. We learned a lot about our patients from these encounters. Appointments in cars became a frequent way for patients, and clinicians, to maintain therapeutic space. We continue to wonder how this shift in frame impacts the ongoing treatment relationship. While we assessed privacy and digital health concerns with each patient, the need for new scales and tools to quantify and assess privacy issues in digital health is necessary to guide the field towards more informed, secure, and safer care.

In general, our patients in established relationships maintained strong therapeutic alliances in this new frame, as evidenced by the decrease in cancellations. While some patients described being uncomfortable speaking openly from their home, even out of earshot, most appeared at ease in their own environment, allowing the treatment to continue uninterrupted. To ensure all patients could benefit from the technology, we developed an administrative role of virtual navigator-related to a digital navigator (Wisniewski \& Torous, 2020)-to reach out to patients for "practice" on the video platform prior to the appointment. This proved helpful to patients and clinicians alike, as clinicians could then use the time for treatment-instead of providing technology assistance. While some have written about very negative experiences, this was not our experience (Markowitz et al. 2020). Our quality metrics from the clinic noted that $92 \%$ of patients felt visits during COVID-19 met their clinical needs. As the types of technology and skills necessary to access it continue to change, finding scales that are easily able to assess digital literacy, telepsychiatry satisfaction, and new forms of therapeutic alliances will be critical to creating standards and benchmarks for the field.

Over this past year we have grown more comfortable with telehealth platforms and technology. All of our clinicians learned to navigate video platforms, regardless of their age. We discovered that there was no one ideal platform, instead utilizing five different telehealth platforms to best meet both our clinicians' and patients' needs. We did not formally assess individual clinicians' acceptance or ease with the Telehealth. Anecdotally, our trainees and younger attendings who had "grown up" with technology were more adept with the platforms. We established one of our technology savvy clinicians as the "go to" person for those staff who were struggling to implement the technology and integrate it into their practices, which proved invaluable.

Psychotherapy sessions with established patients were the easiest to transition to video or telephone. Initial psychiatric consultations were the most challenging, specifically with 
acutely ill and cognitively compromised patients. Psychopharmacology follow up meetings were somewhere between the two, depending on the acuity of the patients' symptoms. Using technologies like online forms and apps to collect symptoms was of benefit, especially in our neuropsychology service. We recognize that technology will continue to update and evolve, but regardless of these changes see a role of a core set of digital health clinical competencies that can ensure all clinicians are ready to utilize different modes of technology to reach patients in needed.

We faced several challenges that went beyond privacy, alliance, and technology. While almost all patients were interested and willing to use technology, socioeconomic status limited some access to the internet, video, or telephone. Linking different technology systems together, for example remote surveys appearing in medical records, was a challenge as was capturing data around gait, weight, and the Abnormal Involuntary Movement Scale (AIMS) as three examples. Finally, continued concerns around the pandemic limited the number of labs patients were willing to obtain.

As a teaching hospital, one of our missions is to provide excellent clinical training of psychiatry residents. In our program, although each second year resident treats one outpatient, our residents' primary exposure to outpatient work is during their third and fourth years when they spend almost half of their time in our clinic. It is striking to reflect that our third year residents who began the year in July 2020, have been learning outpatient psychiatry primarily from their kitchens and living rooms. While our experienced clinicians struggled to adjust to Telehealth, their vast clinical experience was still brought to the work. Most of our third year residents have never met their patients in person. Concepts such as the psychiatric frame have been learned in the new adjusted format. Supervision has been adapted to video platforms, and quick consultations are accessed via Facetime or phone calls. The ease of walking into a colleague's office to share an important "moment" in treatment-joyful or troubling, has been lost. Zoom didactics and seminars have been shortened due to fatigue. Clinical case conferences have been adapted to patients joining via the video platform for the interview. It is unclear what aspects of learning have been lost, while what else has been gained.

\section{Next Steps}

The future of telehealth integration in outpatient practice will be determined by multiple factors (Haque, 2021). These factors present challenging confounders to ongoing and new research efforts. First, all outpatient treatment is not alike. The nature of the practice as well as the unique needs of a patient population must be considered. Our experience suggests that a hybrid model of in person and video may serve the needs of most established stable patients, balancing telehealth appointments with intermittent in person visits. Initial evaluations and urgent clinical matters are likely best served with in person visits to provide a more thorough assessment. Second, reimbursement parity for telehealth around COVD-19 facilitated this transition to remote. Although the governor of our state passed a law safeguarding telehealth coverage for the next two years, it will require the cooperation of insurers and regulatory bodies. Understanding new reimbursement models is critical at this juncture for understanding what forms of care will be feasible to offer. Finally, we believe that technology and telehealth options will continue to expand and pose new opportunities for enhanced care. Exploring how we can ensure those with lower digital literacy, cognitive impairment, and less access to stable internet is an important consideration in ensuring equality to care. Understanding and accounting for these factors in new research efforts will be an equally important consideration in understanding the impact and effect of telehealth in the coming year.

Areas for future study abound and have only just begun. Just as COVID-19 offers the chance to now rethink the public mental health system (Smith et al., 2021), it also presents the same opportunity at the clinic level with concomitant questions. Preliminary studies suggest that while many patients have reported their experience to be positive with Telehealth, a significant group prefers to return to in person. This was found in own our patient satisfaction survey results as well. One study found that approximately $8 \%$ of participants report that they would continue seeing their mental health provider by video after the stay at home recommendations are lifted (Costa et al., 2020). A more robust study found that a little over half of patients surveyed agreed they would consider remote treatment in the future (Guinart et al., 2021). Understanding the actual needs and preferences of patients, as well as clinicians, is a simple first step but one for which a clear picture does not actually exist today. As mental health services continue to adopt, new technologies will likely continue to accelerate change (Bickman 2020).

\section{Conclusion}

On both the systemic and individual level, we are left with a fair amount of uncertainty of what outpatient practice will look like at the end of 2021 and beyond. While research was harder during 2020, it also taught us flexibility in the face of crisis can provide opportunities. Telehealth enabled us to care for our patients through a period of intense instability and loss. After the pandemic passes, with renewed research 
efforts we will discover whether telehealth offers not only adequate care, but enhanced care.

Author contribution All authors contributed equally.

\section{Declarations}

Conflict of interests The authors declare that they have no conflict of interests.

\section{References}

Bickman, L. (2020). Improving mental health services: A 50-year journey from randomized experiments to artificial intelligence and precision mental health. Administration and Policy in Mental Health and Mental Health Services Research, 47(5), 795-843.

Costa, M., Reis, G., Pavlo, A., Bellamy, C., Ponte, K., \& Davidson, L. (2020). Tele-mental health utilization among people with mental illness to access care during the CoViD-19 pandemic. Community Mental Health Journal, 57(4), 720-726.

Guinart, D., Marcy, P., Hauser, M., Dwyer, M., \& Kane, J. (2020). Patient attitudes toward telepsychiatry during the COVID-19 pandemic: A nationwide, multisite survey. JMIR Mental Health, 7(12), e24761.
Haque, S. N. (2021). Telehealth beyond COVID-19. Psychiatric Services, 72(1), 100-103.

Markowitz, J. C., Milrod, B., Heckman, T. G., Bergman, M., Amsalem, D., Zalman, H., Ballas, T., \& Neria, Y. (2020). Psychotherapy at a distance. American Journal of Psychiatry. https://doi.org/10.1176/ appi.ajp.2020.20050557

Smith, T. E., Sullivan, A. M. T., \& Druss, B. (2021). Redesigning public mental health systems post: COVID-19. Psychiatric Services, 72(5), 602-605.

Uscher-Pines, L., Sousa, J., Raja, P., Mehrotra, A., Barnett, M. L., \& Huskamp, H. A. (2020). Suddenly becoming a "virtual doctor": Experiences of psychiatrists transitioning to telemedicine during the COVID-19 pandemic. Psychiatric Services, 71(11), 1143-1150.

Wisniewski, H., \& Torous, J. (2020). Digital navigators to implement smartphone and digital tools in care. Acta Psychiatrica Scandinavica, 141(4), 350-355.

Yellowlees, P., Nakagawa, K., Pakyurek, M., Hanson, A., Elder, J., \& Kales, H. C. (2020). Rapid conversion of an outpatient psychiatric clinic to a $100 \%$ virtual telepsychiatry clinic in response to COVID-19. Psychiatric Services, 71(7), 749-752.

Publisher's Note Springer Nature remains neutral with regard to jurisdictional claims in published maps and institutional affiliations. 